\title{
Estimation of transverse spin penetration length using second-harmonic measurement: Proposal of an experimental method
}

\author{
Pavel Baláž, ${ }^{1, *}$ Maciej Zwierzycki, ${ }^{2}$ Jean-Philippe Ansermet, ${ }^{3}$ and Józef Barnaś ${ }^{2,4}$ \\ ${ }^{1}$ Charles University, Faculty of Mathematics and Physics, Department of Condensed Matter Physics, \\ Ke Karlovu 5, 12116 Prague, Czech Republic \\ ${ }^{2}$ Institute of Molecular Physics, Polish Academy of Sciences, Smoluchowskiego 17, 60-179 Poznań, Poland \\ ${ }^{3}$ Ecole Polytechnique Fédérale de Lausanne, IPMC-Station 3, CH-1015 Lausanne-EPFL, Switzerland \\ ${ }^{4}$ Adam Mickiewicz University, Faculty of Physics, Umultowska 85, 61-614 Poznań, Poland \\ (Received 29 July 2016; revised manuscript received 22 September 2016; published 13 October 2016)
}

\begin{abstract}
A theoretical description of spin current injection from a nonmagnetic layer into a magnetic one is presented, with the main emphasis on the description and determination of the penetration depth of spin current component transverse to the magnetization. This penetration depth also determines the depth of spin transfer torque generation. Physically, the spin current may be driven by an external electric field or by a temperature gradient. To determine the penetration depth we used ab initio calculations of channel and mixing conductances as well as of mixing transmission. The results are then used to determine the second harmonic voltage response, which in turn can be used to determine the penetration depth experimentally.
\end{abstract}

DOI: 10.1103/PhysRevB.94.144414

\section{INTRODUCTION}

During the last decade, spin transfer torque (STT) [1-4] became a well established notion in modern physics, with a clear interpretation: mobile electronic carriers transfer part of their spin angular momentum to collective magnetic degrees of freedom of a magnetic object, leading to a change in its magnetic state. The main motivation to investigate this phenomenon is opening new ways of rapid manipulation of localized magnetic moments at low energy costs $[5,6]$. To find systems of high efficiency of spin transfer, the spin torque was studied in various experimental setups ranging from current-in-plane (CIP) [7,8] or current-perpendicular-to-plane (CPP) [9] spin valves consisting of ferromagnetic (FM) layers separated by nonmagnetic spacers up to more recent devices made of a ferromagnetic layer on top of a heavy metal with strong intrinsic spin-orbit coupling and utilizing the spin Hall effect to generate spin current at the corresponding interface [10]. The spin current, and thus also the spin transfer torque, can be generated by an external electric field, temperature gradient owing to thermoelectric and/or thermospin phenomena [11-13], spin pumping [14], as well as by nonequilibrium laser-induced excitation of hot electrons [15].

Although the number of ferromagnetic elements became smaller in new devices, the mechanism of spin transfer to ferromagnetic metals still remains an open question. This applies especially to the transverse spin penetration length (TSPL): a length at which spin components perpendicular to magnetization direction can penetrate into a magnetic layer. The question of TSPL becomes important in devices with magnetic layers as thin as a few lattice constants. A commonly accepted assumption is that transverse spin current components are absorbed directly at the nonmagnet/ferromagnet (N/FM) interface. Indeed, in most recent experimental studies it has been concluded that the TSPL is short and the spin current is almost completely absorbed in the first few atomic layers

*balaz@karlov.mff.cuni.cz from the interface [16]. In contrast, from another experimental research it follows that the TSPL can be as large as a few nanometers, depending on the material $[17,18]$.

The above mentioned experiments based on a spin pumping [14] method utilized FM1/N/FM2 systems consisting of two magnetic layers of different materials separated by a nonmagnetic spacer. Both magnetic materials should have different resonance frequencies in order to excite magnetic dynamics in one of them. The precessing magnetization of, e.g., the FM1 layer pumps transverse spin current to the adjacent nonmagnetic $(\mathrm{N})$ layer creating in it a spin accumulation. The second magnetic layer, FM2, acts then as a spin sink. In the experiment, thickness of the FM2 layer was increased and the power absorption spectra of the bilayer, which is proportional to the Gilbert damping rate, was measured. In Refs. [17,18] the authors used the model by Zhang, Levy, and Fert $[19,20]$, which describes transport of the transverse spin current components in terms of the diffusion equation, with TSPL being a free parameter which can be estimated by fitting theoretical results to experimentally obtained ones. On the contrary, Ghosh et al. [16] employed the magnetoelectronic circuit theory [21] relating the Gilbert damping to the effective spin mixing conductance of the magnetic trilayer. The TSPL of FM2 layer was then estimated as the thickness at which the Gilbert damping saturates.

In the experimental setup described above, two problems might arise: (i) The diffusive description used by Taniguchi et al. $[17,18]$ is restricted to weak magnets as in the strong ferromagnetic metals the quantum decoherence dominates the decay of transverse spin components [22,23]. Moreover, if the assumption of a very small TSPL is correct, a diffusive model is not valid for extremely thin magnetic layers. In this case magnetoelectronic circuit theory provides a correct description assuming the whole magnetic layer as a magnetic scatterer embedded in a diffusive environment described as an interface with spin-dependent reflection and transmission coefficients. (ii) The description of Ghosh et al. [16] is based on the effective spin mixing conductance. In the case of thick magnetic layers, the real part of mixing conductance dominates 
transport through the FM2 layer. However, in the case of smaller thickness of FM2, also the imaginary part of the mixing conductance might be important. Moreover, the spin mixing conductance depends on the properties of N/FM2 interface and it relaxes provided the FM2 thickness exceeds a few atomic layers. However, in the case of thin magnetic layers, there is a chance that some transverse spins can pass the magnetic film and build up transverse spin accumulation in the nonmagnetic lead. This process is described by the mixing transmission which depends on the whole FM2 layer.

The main objectives of this paper are as follows. First, we show that the second harmonic voltage measurements can be used to estimate the out-of-plane STT component. Since the magnitude of STT components depends on the spin mixing quantities of the magnetic layer, the second harmonic voltage gives us access to some of them, namely to the difference of imaginary parts of the mixing conductance and mixing transmission. Second, we properly define TSPL in terms of basic transport parameters. Finally, we provide an experimental method to measure TSPL, which might serve as a complementary scheme to the above mentioned one based on the spin pumping phenomenon. In order to describe consistently the process of spin absorption, we employed in this paper $a b$ initio calculations. Using the wave function matching method, we calculated the channel and mixing conductances as well as the mixing transmissions as a function of layer thickness. This step allowed us to properly define the TSPL. Consequently, in the second step we assume the magnetic layer is embedded as a free layer in a dual spin valve structure. Analyzing the magnetoresistance of the spin valve and spin torque exerted on the free magnetic layer we suggest an experimental method to measure TSPL in magnetic conductors. The method is based on the analysis of angular dependence of the second harmonic voltage response to the spin current [23-26]. To demonstrate all features of the method, we have chosen some of the most typical combinations of magnetic and nonmagnetic materials used for fabrication of metallic spin valves: $\mathrm{Cu} / \mathrm{Co}, \mathrm{Cu} / \mathrm{Ni}$, and $\mathrm{Au} / \mathrm{Fe}$.

The paper is organized as follows. In Sec. II we introduce the theoretical models we used, especially the ab initio method employed to calculate the interface conductances and mixing transmission. Then we show how they can be incorporated into the diffusive transport model. Calculation of the second harmonic voltage is also explained there. In Sec. III we present and analyze our numerical results. In this section we analyze a $\mathrm{Cu} / \mathrm{Co}$ spin valve in more detail. First, the results of ab initio calculations are described, and then we analyze the calculated STT components and magnetoresistance as a function of the free layer thickness. Finally, we calculate the second harmonic voltage which is compared to ab initio calculated imaginary parts of the spin mixing parameters. In case of other spin valves, we present just the dependence of the second harmonic voltage and the spin mixing parameters on the layer thickness. A summary of the obtained results and final conclusions are presented in Sec. IV.

\section{THEORETICAL MODELS}

The spin valve structure considered in this paper is shown schematically in Fig. 1(a). It consists of a thin magnetic

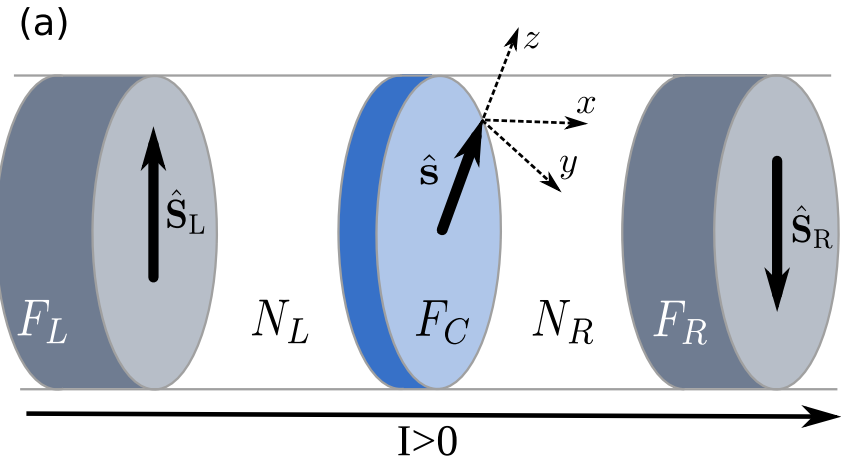

(b)

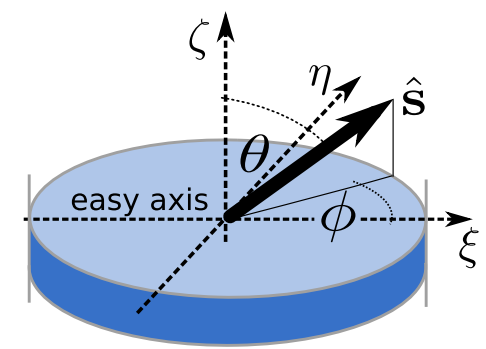

FIG. 1. (a) Model of a dual spin valve structure assumed in this paper. $F_{L}$ and $F_{R}$ are the fixed magnetic layers with opposite magnetization directions, $\mathrm{N}_{\mathrm{L}}$ and $\mathrm{N}_{\mathrm{R}}$ are nonmagnetic spacers, and $\mathrm{F}_{\mathrm{C}}$ is the free magnetic layer. The local coordinate system $(x, y, z)$ in the free layer is also shown there. This coordinate system is used in the description of spin transport and calculation of spin transfer torques. (b) Spherical coordinates $\theta$ and $\phi$ are used in this paper for description of magnetic dynamics. Here the axis $\xi$ is along the magnetic easy axis, while the axis $\zeta$ is normal to the layer.

layer which is separated from two external magnetic films by nonmagnetic spacers. The external magnetic films are assumed to have fixed magnetic moments. Unit vectors along the spin moments of the central and external magnetic layers are denoted as $\mathbf{s}, \mathbf{S}_{\mathrm{L}}$, and $\mathbf{S}_{\mathrm{R}}$, respectively. In equilibrium situation magnetic moments of all three layers are oriented in the corresponding film planes and also along a common easy axis. However, magnetic moments of the external magnetic films are assumed to be antiparallel as shown in Fig. 1(a), where also the coordinate system used in the description of spin transport and spin torques is shown.

Bearing in mind the main objective of the paper, which is to calculate the second harmonic voltage as a linear response of the system to a low-frequency current, we calculate first transport parameters of the thin central magnetic layer. This layer is treated as a magnetic scatterer, and scattering is described by the corresponding transmission and reflection amplitudes as shown schematically in Fig. 2. These scattering amplitudes determine transport parameters of the thin magnetic layer, i.e., channel and mixing conductances as well as mixing transmission, which are calculated using $a b$ initio methods. Then we assume an external magnetic field oriented in the film plane at an angle $\Theta$ with respect to the easy axis, which tilts magnetic moment of the central layer away from the easy axis, as indicated in Fig. 1(a). In the next step we calculate spin torque due to a low-frequency current. Since magnetic dynamics of the central layer around the corresponding equilibrium position (which is in the layer 


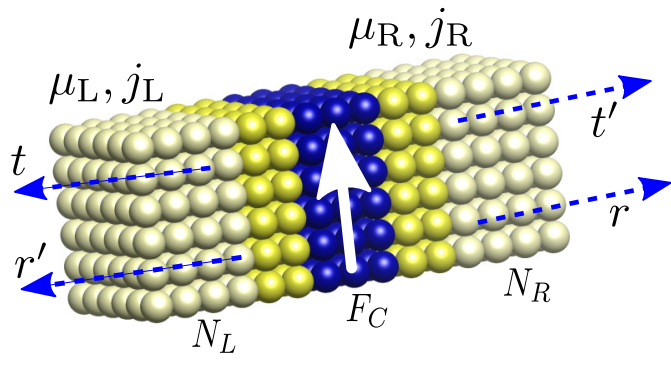

FIG. 2. Scheme of a magnetic scatterer. The central area (blue balls) is the central magnetic layer with uniform magnetization (white arrow). Together with the interface atomic monolayers of the adjacent nonmagnets (yellow balls) it becomes the scatterer described by the transmission and reflection coefficients. The parameters $r$ and $t$ are the reflection and transmission amplitudes for electrons moving from the right nonmagnetic lead, while $r^{\prime}$ and $t^{\prime}$ describe reflection and transmission amplitudes for electrons incident from the left nonmagnetic lead. For clarity we skipped the channel indices $n$ and $n^{\prime}$. The blue arrows in the leads show directions of reflected/transmitted electrons related to the mentioned coefficients. In a symmetric case, assumed in this paper, $r=r^{\prime}$ and $t=t^{\prime}$.

plane) will be considered in the linear response regime, it is sufficient to calculate the spin torques in the configuration shown in Fig. 1(a), i.e., when all magnetic moments are in the layer plane.

\section{A. Ab initio calculations}

To obtain transport parameters of an ultrathin magnetic layer we first performed $a b$ initio calculations of the transmission/reflection amplitudes. To do this, the local spin density approximation (LSDA) of the density functional theory (DFT) was employed in a two-step procedure. First, the self-consistent potentials for magnetic layer $(\mathrm{Co}, \mathrm{Ni}, \mathrm{Fe})$ sandwiched between ideal nonmagnetic $(\mathrm{Cu}, \mathrm{Au})$ leads were determined using the surface Green's function formulation of the tight-binding linear muffin tin orbital (TB-LMTO) method $[27,28]$ in atomic-sphere approximation (ASA). Perfect lattice match was assumed in all the cases, with atoms occupying the sites of their respective lattices. Apart from this, translational invariance in the lateral directions was assumed. A minimal $s p d$ basis set was used for all calculations, and the determined atomic-sphere potentials were subsequently used to calculate scattering coefficients by means of the wave function matching method as described in Refs. [29,30].

To include more realistic effects in our model, we studied also the effect of substitutional disorder, which might arise at an interface between two materials. The disorder was modeled using $20 \times 20$ lateral supercells. In this case, the coherent potential approximation (CPA) [28], which yields separate atomic-sphere potentials for intermixed species, was used in the first step of the procedure. The atomic-sphere potentials were then distributed randomly within the supercell in required concentrations. In the following, we shall adopt the standard model of the disordered magnetic layer ( $N$ monolayers thick) as consisting of $N-1$ clean monolayers sandwiched between two monolayers of $50 \%$ alloy. Finally, the summation over the two-dimensional Brillouin zone (2D BZ) was performed using sampling densities corresponding to $10^{3}-10^{4} \mathbf{k}_{||}$points in the $2 \mathrm{D} \mathrm{BZ}$ of the $1 \times 1$ interface unit cell.

From the $a b$ initio calculations we obtain the conductances per unit area for spin-up $G_{\uparrow}$ and spin-down $G_{\downarrow}$ channels, real and imaginary parts of the mixing conductance $G_{\uparrow \downarrow}$, and mixing transmission $T_{\uparrow \downarrow}$. These quantities are related to the spin transmission $t_{\uparrow(\downarrow)}$ and reflection $r_{\uparrow(\downarrow)}$ amplitudes, see Fig. 2. Generally they are defined as [31]

$$
\begin{aligned}
G_{\uparrow(\downarrow)} & =\frac{1}{S} \frac{e^{2}}{h} \sum_{n n^{\prime}}\left[\delta_{n n^{\prime}}-r_{n n^{\prime}}^{\uparrow(\downarrow)}\left(r_{n n^{\prime}}^{\uparrow(\downarrow)}\right)^{*}\right], \\
G_{\uparrow \downarrow} & =\frac{1}{S} \frac{e^{2}}{h} \sum_{n n^{\prime}}\left[\delta_{n n^{\prime}}-r_{n n^{\prime}}^{\uparrow}\left(r_{n n^{\prime}}^{\downarrow}\right)^{*}\right], \\
T_{\uparrow \downarrow} & =\frac{1}{S} \frac{e^{2}}{h} \sum_{n n^{\prime}} t_{n n^{\prime}}^{\uparrow}\left(t_{n n^{\prime}}^{\downarrow}\right)^{*},
\end{aligned}
$$

where $S$ is the area of the layered system, while $n$ and $n^{\prime}$ run over all channels in the relevant nonmagnetic lead. In a general case, the transmission and reflection coefficients for electrons moving from right to left (described by $r_{n n^{\prime}}$ and $t_{n n^{\prime}}$ ) and from left to right (described by $r_{n n^{\prime}}^{\prime}$ and $t_{n n^{\prime}}^{\prime}$ ) may differ, as shown in Fig. 2. In this paper, however, we assume a symmetric scatterer, for which $r_{n n^{\prime}}=r_{n n^{\prime}}^{\prime}$ and $t_{n n^{\prime}}=t_{n n^{\prime}}^{\prime}$.

In order to avoid any unphysical contact resistance, the obtained conductances have to be renormalized according to the formulas [21]

$$
\frac{1}{\tilde{G}_{\uparrow(\downarrow)}}=\frac{1}{G_{\uparrow(\downarrow)}}-\frac{1}{G_{\mathrm{Sh}}},
$$

for the channel conductances, where $G_{\text {Sh }}$ is the Sharvin conductance for the spin channel, and

$$
\begin{aligned}
\frac{1}{\tilde{G}_{\uparrow \downarrow}} & =\frac{1}{G_{\uparrow \downarrow}+T_{\uparrow \downarrow}^{2} /\left(2 G_{\mathrm{Sh}}-G_{\uparrow \downarrow}\right)}-\frac{1}{2 G_{\mathrm{Sh}}}, \\
\frac{1}{\tilde{T}_{\uparrow \downarrow}} & =\frac{\left(2 G_{\mathrm{Sh}}-G_{\uparrow \downarrow}\right)^{2} / T_{\uparrow \downarrow}-T_{\uparrow \downarrow}}{4 G_{\mathrm{Sh}}^{2}},
\end{aligned}
$$

for the spin mixing parameters, which are generally complex numbers $\tilde{G}_{\uparrow \downarrow}=\tilde{g}_{\mathrm{r}}+i \tilde{g}_{\mathrm{i}}$ and $\tilde{T}_{\uparrow \downarrow}=\tilde{t}_{\mathrm{r}}+i \tilde{t}_{\mathrm{i}}$. Although in the following text we will skip, for the sake of the clarity, the tilde notation of the renormalized transport parameters, all these parameters discussed in the subsequent analysis will be renormalized according to Eqs. (2) and (3).

\section{B. Transport through an ultrathin free layer}

As stated above, we consider a magnetic free layer sandwiched between two nonmagnetic spacers attached to thick magnetic polarizers (fixed layers). Since the free magnetic layer is generally thin in comparison to the spin diffusion length, we consider it as a magnetic scatterer described by spin channel conductances, mixing conductance, and mixing transmission. Note, in the system under consideration, the magnetic scatterer includes not only the magnetic layer but also its closest $\mathrm{Cu}$ atomic layers with an atomic potential different from the corresponding bulk ones, see Fig. 2.

Assume that a positive charge current flows from left to right. The transverse spin currents [with respect to the 
magnetization direction in $F_{c}$, see Fig. 1(a)] on the left (L) and right $(\mathrm{R})$ side of the scatterer,

$$
j_{\perp \mathrm{L}}=\left(\begin{array}{c}
j_{x \mathrm{~L}} \\
j_{y \mathrm{~L}}
\end{array}\right) \quad \text { and } \quad j_{\perp \mathrm{R}}=\left(\begin{array}{c}
j_{x \mathrm{R}} \\
j_{y \mathrm{R}}
\end{array}\right)
$$

are related to the transverse spin accumulations

$$
\boldsymbol{\mu}_{\perp \mathrm{L}}=\left(\begin{array}{c}
\mu_{x \mathrm{~L}} \\
\mu_{y \mathrm{~L}}
\end{array}\right) \quad \text { and } \quad \boldsymbol{\mu}_{\perp \mathrm{R}}=\left(\begin{array}{c}
\mu_{x \mathrm{R}} \\
\mu_{y \mathrm{R}}
\end{array}\right)
$$

as follows (assuming symmetric magnetic scatterer):

$$
e^{2}\left(\begin{array}{l}
\boldsymbol{j}_{\perp \mathrm{R}} \\
\boldsymbol{j}_{\perp \mathrm{L}}
\end{array}\right)=\left(\begin{array}{cc}
\boldsymbol{G} & \boldsymbol{T} \\
-\boldsymbol{T} & -\boldsymbol{G}
\end{array}\right)\left(\begin{array}{l}
\boldsymbol{\mu}_{\perp \mathrm{R}} \\
\boldsymbol{\mu}_{\perp \mathrm{L}}
\end{array}\right)
$$

where

$$
\boldsymbol{G}=2\left(\begin{array}{cc}
-g_{\mathrm{r}} & g_{\mathrm{i}} \\
-g_{\mathrm{i}} & -g_{\mathrm{r}}
\end{array}\right) \quad \text { and } \quad \boldsymbol{T}=2\left(\begin{array}{cc}
t_{\mathrm{r}} & -t_{\mathrm{i}} \\
t_{\mathrm{i}} & t_{\mathrm{r}}
\end{array}\right) .
$$

Equation (6) represents boundary conditions which allow us to introduce an ultrathin magnetic layer into a diffusive environment. Thus, the rest of the spin valve structure shall be divided into bulk layers separated by interfaces, and treated in frame of the model described in Ref. [32], which is valid for spin transport in noncollinear spin valve structures.

The spin transfer torque is defined as

$$
\boldsymbol{\tau}=\frac{\hbar}{2}\left(\boldsymbol{j}_{\perp \mathrm{L}}-\boldsymbol{j}_{\perp \mathrm{R}}\right) .
$$

Generally, $\boldsymbol{\tau}$ can be decomposed into two parts; the in-plane (antidamping) torque $\boldsymbol{\tau}_{\|}$, and the out-of-plane (fieldlike) torque. Assuming antiparallel orientation of the magnetic moments of the outermost magnetic layers, $\hat{S}_{\mathrm{L}}=-\hat{\boldsymbol{S}}_{\mathrm{R}} \equiv \hat{\boldsymbol{S}}$, one can write [33]

$$
\begin{aligned}
\boldsymbol{\tau}_{\|} & =I a \hat{\boldsymbol{s}} \times(\hat{\boldsymbol{s}} \times \hat{\boldsymbol{S}}), \\
\boldsymbol{\tau}_{\perp} & =I b \hat{\boldsymbol{s}} \times \hat{\boldsymbol{S}},
\end{aligned}
$$

where $I$ is the charge current density, while

$$
\begin{aligned}
a & =-\frac{\hbar}{2 I} \frac{j_{y \mathrm{~L}}-j_{y \mathrm{R}}}{\sin \phi}, \\
b & =\frac{\hbar}{2 I} \frac{j_{x \mathrm{~L}}-j_{x \mathrm{R}}}{\sin \phi},
\end{aligned}
$$

with $\phi$ being an angle between $\hat{\boldsymbol{s}}$ and $\hat{\boldsymbol{S}}$ (we remind that in this section both $\hat{\boldsymbol{s}}$ and $\hat{\boldsymbol{S}}$ are in the layer plane). The latter expressions can be rewritten as functions of spin accumulation

$$
\begin{aligned}
& a=-\frac{\hbar}{e^{2} I} \frac{a_{\mathrm{r}} \mu_{y}+a_{\mathrm{i}} \mu_{x}}{\sin \phi}, \\
& b=\frac{\hbar}{e^{2} I} \frac{a_{\mathrm{r}} \mu_{x}-a_{\mathrm{i}} \mu_{y}}{\sin \phi},
\end{aligned}
$$

where $a_{\mathrm{r}}$ and $a_{\mathrm{i}}$ are real and imaginary parts of $A_{\uparrow \downarrow}=G_{\uparrow \downarrow}-$ $T_{\uparrow \downarrow}$, respectively. Moreover, $\mu_{x}=\mu_{x \mathrm{~L}}+\mu_{x \mathrm{R}}$ and $\mu_{y}=$ $\mu_{y \mathrm{~L}}+\mu_{y \mathrm{R}}$. Values of these variables have to be determined from the spin currents calculated using the diffusive transport model [32].

Since $\mu_{x} \ll \mu_{y}$, from Eqs. (11) one obtains

$$
a \simeq-a_{\mathrm{r}} \frac{\hbar}{e^{2} I} \frac{\mu_{y}}{\sin \phi} \quad \text { and } \quad b \simeq-a_{\mathrm{i}} \frac{\hbar}{e^{2} I} \frac{\mu_{y}}{\sin \phi},
$$

which implies that the in-plane torque is proportional to the real part of $A_{\uparrow \downarrow}\left(\boldsymbol{\tau}_{\|} \propto a_{\mathrm{r}}\right)$ while the out-of-plane torque is proportional to the imaginary part of $A_{\uparrow \downarrow}\left(\boldsymbol{\tau}_{\perp} \propto a_{\mathrm{i}}\right)$.

\section{Second harmonic voltage response}

One of the methods proposed for experimental determination of TSPL is based on the second harmonic response. The key point of this method is to measure a second harmonic voltage response to low-frequency current flowing through the system. The current creates a spin torque which induces magnetic dynamics of the central layer. Since the current density is small, this dynamics is usually considered in the linear response regime. The induced low-frequency magnetic dynamics modifies accordingly the system resistance due to giant magnetoresistance effect. This, in turn, gives rise to a second harmonic voltage $U_{2 \omega}$ which can be calculated from the formula [23]

$$
U_{2 \omega}=-\left.\frac{I_{0}^{2}}{4} \frac{\partial R}{\partial v}\right|_{\phi_{0}} \sin \phi_{0}\left|\chi_{\phi}\right|,
$$

where $R$ is the total resistance of the spin valve, $v=v(\theta, \phi)=$ $\cos \phi \sin \theta$, and $\chi$ is the susceptibility which reads

$$
\begin{aligned}
& \chi_{\phi}\left(\phi_{0}, \Theta\right) \\
& =-\frac{\left|\gamma_{\mathrm{g}}\right|}{M_{\mathrm{s}} d} \frac{\omega_{1} b_{0}+i\left(a_{0}-\alpha b_{0}\right) \omega}{\left(\alpha \omega_{1}-i \omega\right)\left(\alpha \omega_{2}-i \omega\right)+\omega_{1} \omega_{2}} \sin \phi_{0} .
\end{aligned}
$$

Here $\omega$ is the frequency of the dynamic component of $\hat{\boldsymbol{s}}$, and $\omega_{1}\left(\phi_{0}, \Theta\right)=-\left|\gamma_{\mathrm{g}}\right| \mu_{0}\left[H_{\mathrm{d}}+H_{\mathrm{ani}} \cos ^{2} \Theta+H_{\text {app }} \cos \left(\Theta-\phi_{0}\right)\right]$,

$\omega_{2}\left(\phi_{0}, \Theta\right)=-\left|\gamma_{\mathrm{g}}\right| \mu_{0}\left[H_{\mathrm{ani}} \cos \left(2 \phi_{0}\right)+H_{\mathrm{app}} \cos \left(\Theta-\phi_{0}\right)\right]$.

In the latter equation, $H_{\text {ani }}$ is the uniaxial anisotropy field, and $H_{\mathrm{d}}$ is the dominant out-of-plane component of the selfdemagnetizing field of the free layer. Moreover, $H_{\text {app }}$ is the magnitude of magnetic field, which is applied in the layer's plane at an angle $\Theta$ with respect to the easy axis [axis $\xi$ in Fig. 1(b)],

$$
\mathbf{H}_{\text {app }}=H_{\text {app }}(\cos \Theta, \sin \Theta, 0) .
$$

When defining the spherical coordinates $\phi$ and $\theta$ we used the coordinate system $(\xi, \eta, \zeta)$, see Fig. 1(b). Note the azimuthal angle $\phi$ for $\theta=\pi / 2$ reduces to the angle $\phi$ between magnetic moments used in Sec. II B. In Eqs. (13)-(15), $\phi_{0}$ describes orientation of the spin moment $\hat{s}$ in its equilibrium position under the applied magnetic field. Similarly, $a_{0}=a\left(\phi_{0}\right)$ and $b_{0}=b\left(\phi_{0}\right)$ are the parameters describing the in-plane and out-of-plane STT components, calculated in the equilibrium position of $\hat{\boldsymbol{s}}_{0}$. The stationary points of the dynamics at a given field angle $\Theta$ are determined by the equation

$$
H_{\text {ani }} \sin 2 \phi_{0}-2 H_{\text {app }} \sin \left(\Theta-\phi_{0}\right)=0 .
$$

Note, in the limit of low frequency, $\omega \rightarrow 0$, the susceptibility (14) reduces to

$$
\chi_{\phi}\left(\phi_{0}, \Theta\right)=\frac{\left|\gamma_{\mathrm{g}}\right|}{M_{\mathrm{s}} d} \frac{b_{0}}{\omega_{2}\left(\phi_{0}, \Theta\right)} \sin \phi_{0},
$$


i.e., it is proportional to the imaginary part of $A_{\uparrow \downarrow}$, see Eq. (11). Note that $\theta_{0}=\pi / 2$ and does not occur in the above formulas for the second harmonic response.

\section{NUMERICAL RESULTS}

In this section we shall present numerical results on the second harmonic voltage for double spin valve (DSV) structures consisting of various materials. In the calculations we used the current amplitude of $500 \mu \mathrm{A}$, while for the the Gilbert damping parameter we assumed $\alpha=0.1$. The magnetization of the free layer was set to $M_{\mathrm{s}}=10^{5} \mathrm{~A} / \mathrm{m}$. The demagnetizing field was calculated for a layer of elliptical cross section with the minor and major axes equal to 250 and $400 \mathrm{~nm}$, respectively. For this cross section we also calculated the corresponding current density. In turn, calculation of the susceptibility was done in the low frequency regime, where the susceptibility does not depend on frequency. Thus, we used Eq. (18), which is independent on $\omega$.

\section{A. $\mathrm{Cu} / \mathrm{Co}$ double spin valve}

\section{Clean interfaces}

Consider now some numerical results based on the description presented above, and let us begin with transport parameters of the central magnetic layer. Figure 3 shows the transport parameters of a cobalt magnetic layer attached to copper leads with perfectly ordered interfaces. These parameters are shown as a function of the layer thickness measured by the number of atomic layers. Although some of these parameters were already studied in Ref. [34], we show them all in Fig. 3 in order to explain our motivation to study the second harmonic response voltage. Figures 3(a) and 3(b) show the parameters related to the channel resistances, i.e., $R^{*}$ and $\gamma$, which are defined via the relation $R_{\uparrow(\downarrow)}=2 R^{*}(1 \mp \gamma)$, with $R_{\uparrow(\downarrow)}=1 / G_{\uparrow(\downarrow)}$. Thus, $R^{*}=\left(R_{\uparrow}+R_{\downarrow}\right) / 4$ and $\gamma=$
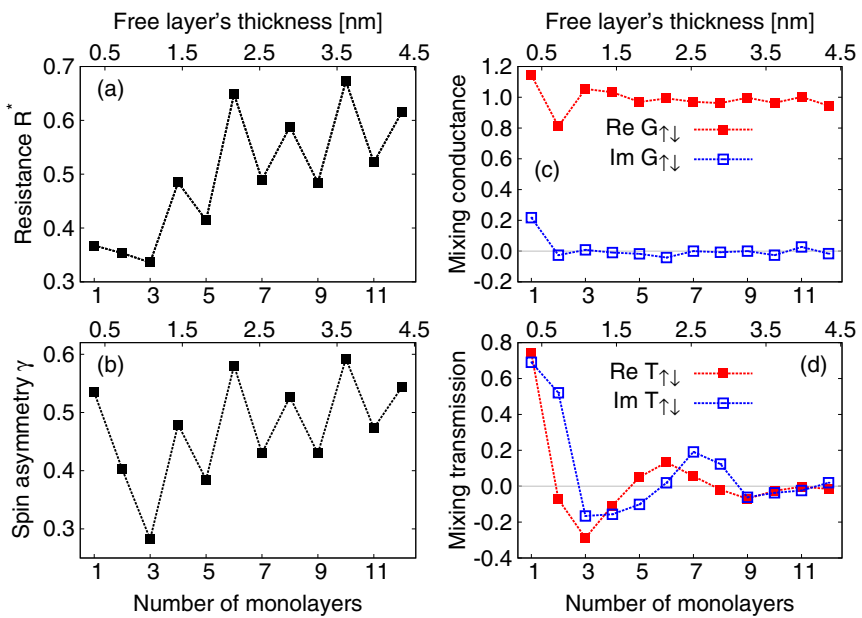

FIG. 3. (a) Resistance $R^{*}$ in the units of $\mathrm{f} \Omega \mathrm{m}^{2}$, (b) the spin asymmetry parameter $\gamma$, (c) real and imaginary parts of the mixing conductance, and (d) real and imaginary parts of the mixing transmission as a function of layer thickness. The mixing conductance and transmission are both in the units of $1 /\left(\mathrm{f} \Omega \mathrm{m}^{2}\right)$. Numerical results are for the $\mathrm{Cu} / \mathrm{Co}(d) / \mathrm{Cu}$ system with clean interfaces $(d$ is the thicknesses of the Co layer).
$\left(R_{\downarrow}-R_{\uparrow}\right) /\left(R_{\uparrow}+R_{\downarrow}\right)$ is the spin asymmetry parameter. Both these quantities oscillate and slightly increase with increasing thickness. On the other hand, Figs. 3(c) and 3(d) show the real and imaginary parts of the mixing conductance $G_{\uparrow \downarrow}$ and mixing transmission $T_{\uparrow \downarrow}$, respectively. From these figures one can make the following observations: (i) Both real and imaginary parts of $G_{\uparrow \downarrow}$ saturate after the number of atomic layers is greater than two. For greater thicknesses these parts do not change except for minor oscillations around the corresponding saturation values, which is in a good agreement with previous calculations [34]. (ii) Variation of $T_{\uparrow \downarrow}$ with the thickness is more pronounced than that of $G_{\uparrow \downarrow}$, and $T_{\uparrow \downarrow}$ reaches relatively high magnitudes up to the thickness of 9 atomic layers. (iii) Comparing the four mixing parameters one can see that the imaginary part of $G_{\uparrow \downarrow}$ is almost zero in the whole range of thicknesses. Thus, its impact on transport is less pronounced. This is in agreement with many experimental and theoretical studies $[34,35]$. Interestingly, the components of the mixing transmission seem to play a more important role than the imaginary part of $G_{\uparrow \downarrow}$. Moreover, in a large range of thicknesses, they remain also comparable to the real part of $G_{\uparrow \downarrow}$. This seems to be the most pronounced difference between the spin transport in ultrathin and standard mesoscopic magnetic layers. In the latter case, transport of the transverse spin components can be satisfactorily described by the real part of the mixing conductance. These observations have an effect on the STT acting on the free magnetic layer and on its current-induced magnetization dynamics, as explained in the following.

Consider now the metallic DSV $\mathrm{Cu}-\mathrm{Co}(8) / \mathrm{Cu}(5) / \mathrm{Co}(d) /$ $\mathrm{Cu}(5) / \mathrm{Co}(8)-\mathrm{Cu}$, where numbers in the brackets are thicknesses of the corresponding layers in nanometers. The outer $\mathrm{Co}(8) \mathrm{FM}$ layers are assumed to have fixed magnetization moments which are aligned in the opposite directions $\left(\hat{S}_{\mathrm{L}}=\right.$ $\left.-\hat{\boldsymbol{S}}_{\mathrm{R}}\right)$, while the central $\operatorname{Co}(d)$ layer is the free magnetic layer with spin magnetic moment $\hat{\boldsymbol{s}}$, see Fig. 1 . In turn, $\mathrm{Cu}(5)$ are nonmagnetic spacer layers, while the outermost $\mathrm{Cu}$ leads play the role of nonmagnetic semi-infinite electrodes. This DSV geometry has been shown to enhance the spin accumulation in the adjacent nonmagnetic layers [33,36]. Since both materials in the fcc phase are nearly ideally lattice matched, a common lattice constant of $3.614 \AA$ was assumed in ab initio calculations. The lattice was oriented so that [111] corresponded to the direction of the current. Note that in this geometry 5 and 8 $\mathrm{nm}$ correspond to 24 and 38 monolayers, respectively.

Figure 4 shows the STT exerted on the Co free magnetic layer. The parameters used for the description of transport in the diffusive part of the spin valve are listed in the Appendix A. Figures 4(a) and 4(b) show the angular dependence of the in-plane (antidamping) and out-of-plane (fieldlike) STT components for several thicknesses of the central Co layer, respectively. Here $N$ is the number of atomic Co monolayers in the free layer. The magnetic moment of the free layer is rotated in the layer's plane $(\theta=\pi / 2)$. The direction of $\hat{s}$ is given by $\phi$, which is the angle between $\hat{\boldsymbol{S}}_{\mathrm{L}}$ and $\hat{\boldsymbol{s}}$. Both components of STT are symmetric with respect to $\phi=\pi / 2$ due to the symmetry of considered DSV. In agreement with other works, the in-plane torque is larger than the fieldlike one for all layer thicknesses. Nevertheless, for $N=1,2$ the difference in the magnitudes of the two STT components is less pronounced than for thicker 

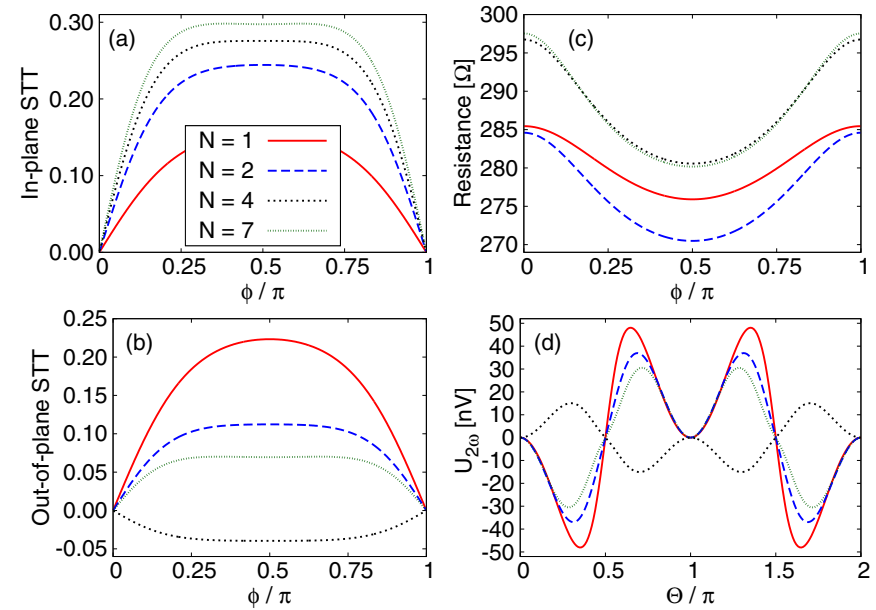

FIG. 4. (a) In-plane and (b) out-of-plane spin torque components as a function of the angle $\phi$ in the units of $\hbar I /|e|$. (c) Resistance of the spin valve as a function of angle $\phi$, and (d) second harmonic voltage as a function of the angle $\Theta$ describing field orientation in the $\mathrm{Cu}-\mathrm{Co}(8) / \mathrm{Cu}(5) / \mathrm{Co}(d) / \mathrm{Cu}(5) / \mathrm{Co}(8)-\mathrm{Cu}$ spin valve with clean interfaces (the numbers in brackets denote thicknesses of the corresponding layers in nanometers).

free layers, where the fieldlike torque is usually negligible [37]. Moreover, one can observe a nonmonotonous variation of both STT components with the layer thickness. These components vary strongly with $N$, especially for small values of $N$. The in-plane component becomes relatively unchanged for $N>3$. This reflects the fact that antidamping torque depends mainly on the real part of the mixing conductance, which is dominant among all mixing parameters shown in Figs. 3(c) and 3(d). Thus, any dependence of the in-plane STT on the imaginary part of $G_{\uparrow \downarrow}$ and on $T_{\uparrow \downarrow}$ does not have remarkable influence on its magnitude as a function of layer thickness. On the contrary, the out-of-plane STT has been shown to depend mainly on the imaginary part of $G_{\uparrow \downarrow}$ [35]. Though the latter approaches zero for $N \geqslant 2$, the out-of-plane torque is still relatively large (and negative) for $N=7$. Thus, one may expect an influence of the spin mixing transmission which rapidly varies with $N$ and does so even at higher values of $N$. Importantly, this feature can be observed also in the angular dependence of the second harmonic voltage shown in Fig. 4(d). This is in good agreement with Eq. (18), which shows that $U_{2 \omega}$ depends mainly on the outof-plane torque component. As a result, one might expect that any dependence of the fieldlike torque on the mixing transmission shall be visible in the measured second harmonic response voltage. Note that Fig. 4(d) plots $U_{2 \omega}$ as a function of $\Theta$, which describes the direction of the external in-plane magnetic field [see Eq. (16)] of magnitude $H_{\text {app }}=3 \mathrm{mT}$. When the current vanishes, $I=0$, the equilibrium position of $\hat{s}$ is generally tilted from the direction of $\mathbf{H}_{\text {app }}$ as described by Eq. (17).

Figure 4(c) shows the angular dependence of the spin valve resistance. Although its magnitude also depends on the free layer thickness, its first derivative does not significantly change with $N$. Therefore, when changing the free layer thickness, variation of the second harmonic voltage [see Eq. (13)] should mostly correspond to variation of the fieldlike STT rather than to the magnetoresistance. Figure 5 presents the central

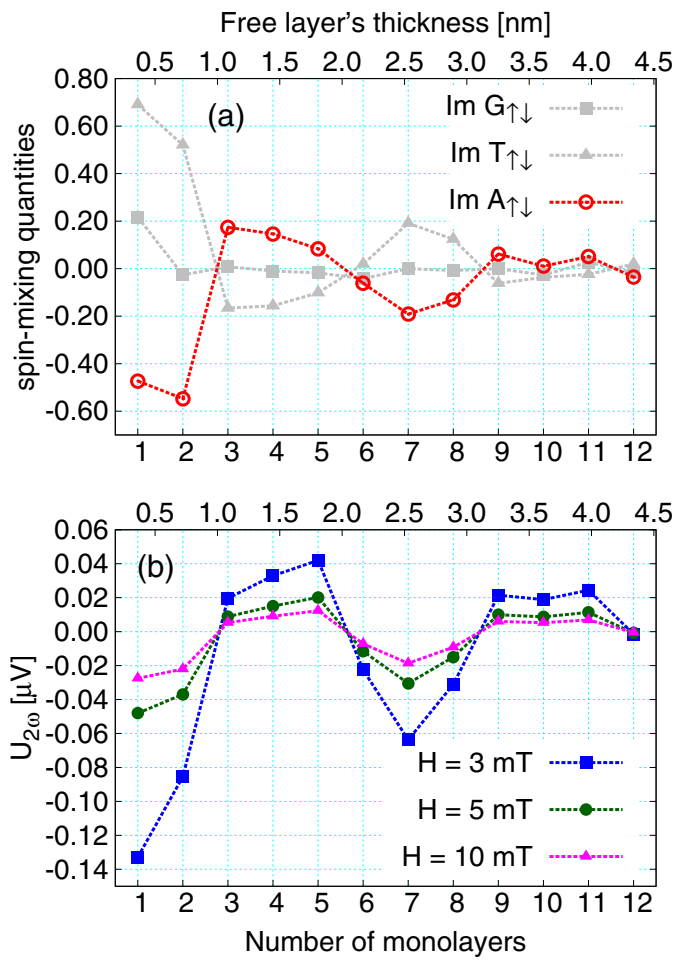

FIG. 5. (a) Imaginary parts of the mixing conductance $G_{\uparrow \downarrow}$, mixing transmission $T_{\uparrow \downarrow}$, and of their difference $A_{\uparrow \downarrow}=G_{\uparrow \downarrow}-T_{\uparrow \downarrow}$ in the units of $1 /\left(\mathrm{f} \Omega \mathrm{m}^{2}\right)$; and (b) magnitude of the second harmonic voltage response as a function of the free layer thickness in the $\mathrm{Cu}-\mathrm{Co}(8) / \mathrm{Cu}(5) / \mathrm{Co}(d) / \mathrm{Cu}(5) / \mathrm{Co}(8)-\mathrm{Cu}$ valve with clean (ordered) interfaces.

point of our analysis. In Fig. 5(a) we show the magnitudes of the imaginary parts of the two spin mixing quantities $G_{\uparrow \downarrow}$ and $T_{\uparrow \downarrow}$, as well as their difference $A_{\uparrow \downarrow}$, as a function of $N$. In turn, Fig. 5(b) shows the corresponding variation of the second harmonic voltage $U_{2 \omega}$. As a representative value of the angular dependence of the second harmonic voltage we have chosen the maximum of $U_{2 \omega}(\Theta)$ for $\Theta$ in the interval $\Theta \in(\pi / 2, \pi)$. We plotted the second harmonic voltage obtained at different magnitudes of the applied magnetic field. Comparing both figures one may notice a qualitative similarity between the curves. Since $\operatorname{Im} A_{\uparrow \downarrow}$ bears an important information on the transverse spin current in the free magnetic layer, the magnitude of $U_{2 \omega}$ not only gives information on the out-of-plane torque, but it also reflects the amount of transverse spin current transmitted into the magnetic layer. One can define the transverse spin penetration length (TSPL) as the thickness of the magnetic layer, where $\operatorname{Im} T_{\uparrow \downarrow}$ approaches zero for the first time. When the oscillations are well resolved and damped exponentially, one can define TSPL via the corresponding exponential law as a distance at which the amplitude decays $e$ times. Thus, in the considered case the corresponding TSPL can be estimated as to be of an order of 6-7 atomic layers.

\section{Effect of interface disorder}

Consider now the effect of substitutional interface disorder $[29,34,38]$ on the second harmonic voltage response and 

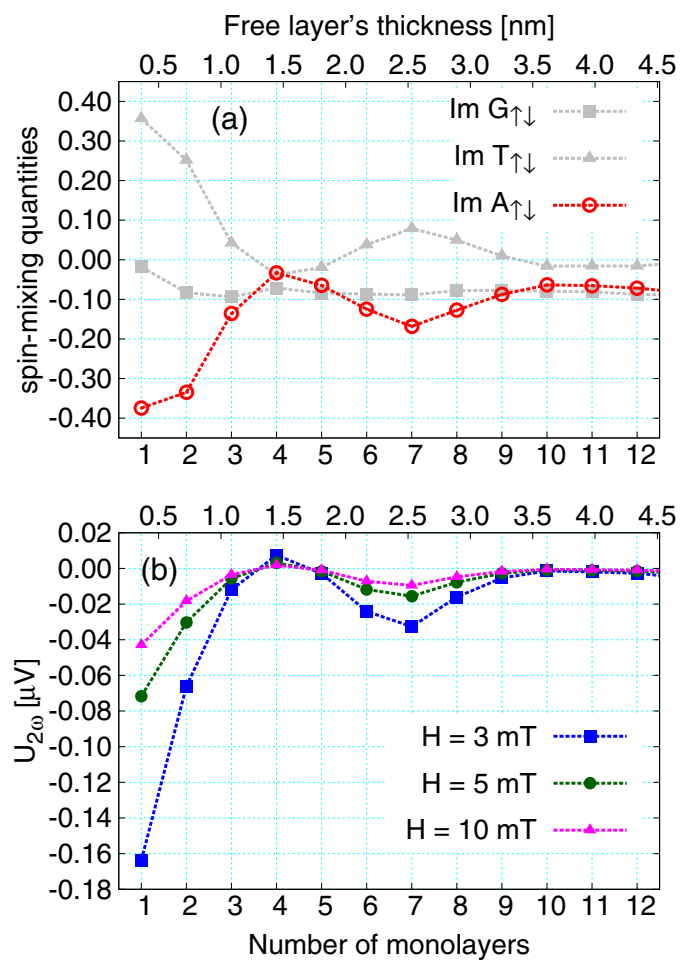

FIG. 6. (a) Imaginary parts of the mixing conductance $G_{\uparrow \downarrow}$, mixing transmission $T_{\uparrow \downarrow}$, and of their difference $A_{\uparrow \downarrow}=G_{\uparrow \downarrow}-T_{\uparrow \downarrow}$ in the units of $1 /\left(\mathrm{f} \Omega \mathrm{m}^{2}\right)$; and (b) magnitude of the second harmonic voltage response as a function of the free layer thickness in the $\mathrm{Cu}-$ $\mathrm{Co}(8) / \mathrm{Cu}(5) / \mathrm{Co}(d) / \mathrm{Cu}(5) / \mathrm{Co}(8)-\mathrm{Cu}$ valve with disordered interfaces of the central magnetic layer.

its relation to the spin mixing transmission parameters. The corresponding channel resistances and mixing parameters for a Co layer with interfacial disorder were calculated using the $a b$ initio methods. Similarly as in the clean situation, we calculated the second harmonic voltage for the $\mathrm{Cu}-\mathrm{Co}(8) / \mathrm{Cu}(5) / \mathrm{Co}(d) / \mathrm{Cu}(5) / \mathrm{Co}(8)-\mathrm{Cu}$ dual spin valve, with the disorder-modified transport parameters of the central magnetic layer. All the other bulk and interface parameters remain the same as in the absence of disorder. Due to the interfacial disorder, both components of the mixing conductance are almost independent of the thickness, with the renormalized values as large as $\operatorname{Re} G_{\uparrow \downarrow} \simeq 1$ and $\operatorname{Im} G_{\uparrow \downarrow} \simeq-0.1$ in the units of $1 /\left(\mathrm{f} \Omega \mathrm{m}^{2}\right)$.

Figure 6(a) shows a strong decrease of $\left|\operatorname{Im} A_{\uparrow \downarrow}\right|$ between $N=1$ and $N=4$. Then, one can observe a minor oscillation of its magnitude for $N>4$. The initial phase of the $\left|\operatorname{Im} A_{\uparrow \downarrow}\right|$ decay is again well reproduced by the maximum of the angular dependence of $U_{2 \omega}$, plotted in Fig. 6(b). Although one finds an overall decrease of $\left|U_{2 \omega}\right|$, for $N \geqslant 5$ one can observe another maximum of $\left|U_{2 \omega}\right|$, which is related to the changes in $\operatorname{Im} A_{\uparrow \downarrow}$. One can notice that the decrease of $\left|\operatorname{Im} A_{\uparrow \downarrow}\right|$ is related to the decrease in $\left|\operatorname{Im} T_{\uparrow \downarrow}\right|$ rather than to the decrease in $\left|\operatorname{Im} G_{\uparrow \downarrow}\right|$ which is roughly a constant function of $N$. Thus, taking into account only mixing conductance $G_{\uparrow \downarrow}$, one would underestimate the total spin penetration length, which in this case is governed by $T_{\uparrow \downarrow}$, and is similar to that in the corresponding clean system.
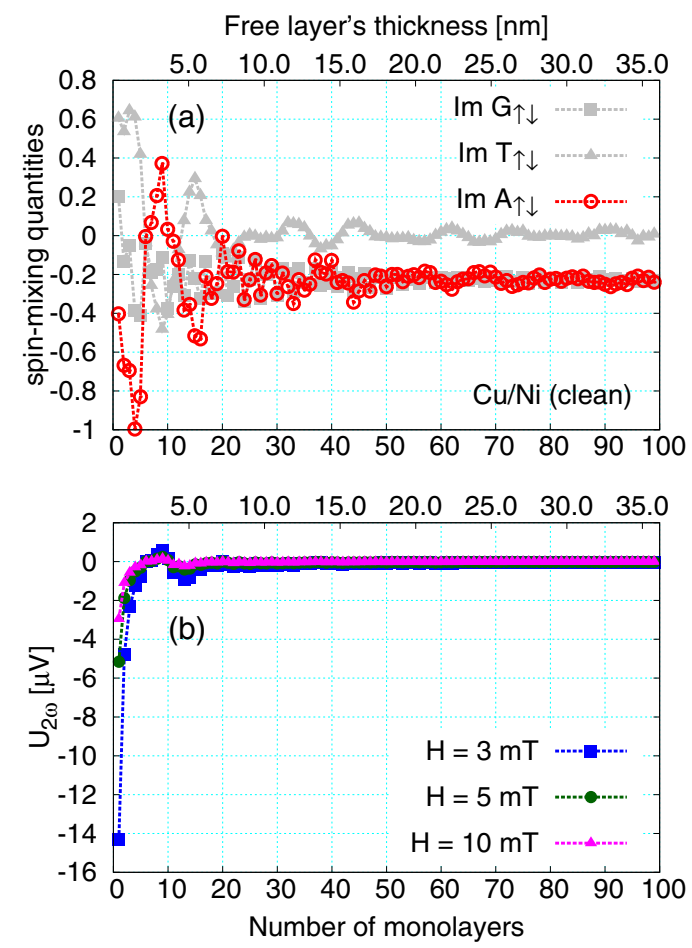

FIG. 7. (a) Imaginary parts of the mixing conductance $G_{\uparrow \downarrow}$, mixing transmission $T_{\uparrow \downarrow}$, and of their difference $A_{\uparrow \downarrow}=G_{\uparrow \downarrow}-T_{\uparrow \downarrow}$ in the units of $1 /\left(\mathrm{f} \Omega \mathrm{m}^{2}\right)$; and (b) magnitude of the second harmonic voltage response as a function of the free layer thickness in the $\mathrm{Cu}-\mathrm{Ni}(8) / \mathrm{Cu}(5) / \mathrm{Ni}(d) / \mathrm{Cu}(5) / \mathrm{Ni}(8)-\mathrm{Cu}$ valve with clean (ordered) interfaces.

\section{B. $\mathrm{Cu} / \mathrm{Ni}$ double spin valve}

Let us now study the second harmonic response in other materials, and focus first on the structure $\mathrm{Cu}-$ $\mathrm{Ni}(8) / \mathrm{Cu}(5) / \mathrm{Ni}(d) / \mathrm{Cu}(5) / \mathrm{Ni}(8)-\mathrm{Cu}$. The lattice constants of $\mathrm{Cu}$ and $\mathrm{Ni}$ as well as the lattice orientation are the same as in the above considered case of $\mathrm{Cu} / \mathrm{Co}$ system. Since the whole methodology is also the same, we show only the main results, i.e., comparison of the imaginary parts of the three mixing parameters with the second harmonic voltage response. This comparison is shown in Fig. 7. One can note that $\operatorname{Im} A_{\uparrow \downarrow}$ shows pronounced oscillations with decreasing amplitude, which persist up to about 100 atomic monolayers. These oscillations appear due to variation of $\operatorname{Im} T_{\uparrow \downarrow}$ with the layer's thickness. In turn, the imaginary part of $G_{\uparrow \downarrow}$ approaches the value of -0.2 . The decrease of $\operatorname{Im} A_{\uparrow \downarrow}$ amplitude is followed by a fast decay of $U_{2 \omega}$. One can also note that the amplitude of $\operatorname{Im} T_{\uparrow \downarrow}$ reduces after $N \simeq 20$. This is also the length on which $U_{2 \omega}$ approaches to values close to zero. Interestingly, the TSPL for Ni layer seems to be an order larger than for Co free layer. Based on $\operatorname{Im} T_{\uparrow \downarrow}$ calculation, it can be estimated to be equal roughly between 20 and 30 atomic planes. This number might be also reckoned from the second harmonic voltage measurement.

Figure 8 shows a comparison for the same system as in Fig. 7, but with interfacial disorder at the central interfaces. The scenario in this case is similar to that in case of $\mathrm{Co} / \mathrm{Cu}$ spin valve with interfacial disorder, shown in Fig. 6. The imaginary part of $G_{\uparrow \downarrow}$ decreases fast, however, amplitude of $\operatorname{Im} T_{\uparrow \downarrow}$ oscillations remain significant at longer distances, 


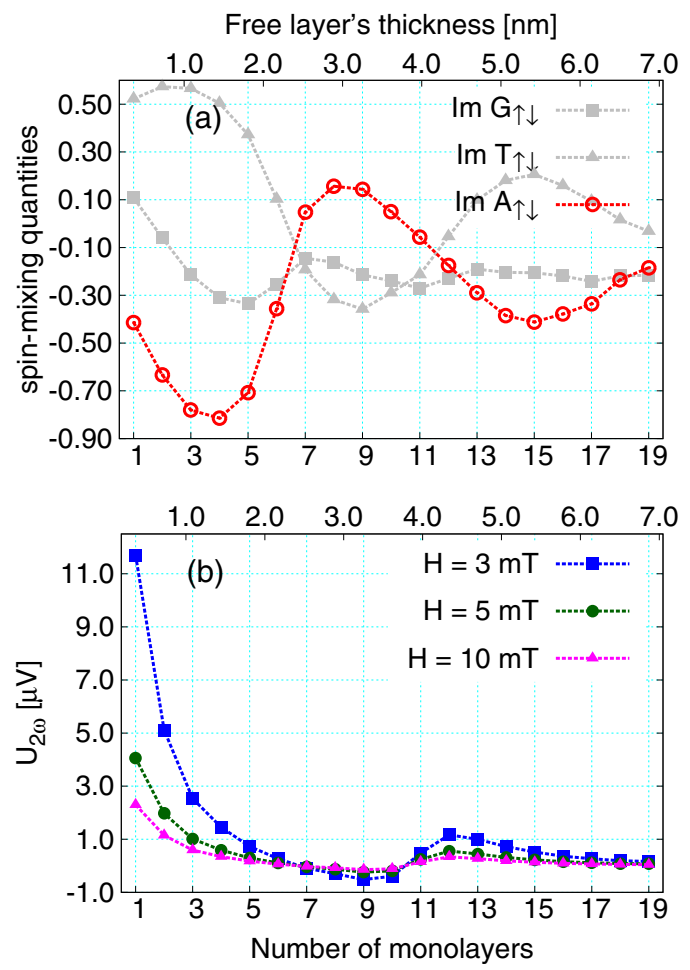

FIG. 8. (a) Imaginary parts of the mixing conductance $G_{\uparrow \downarrow}$, mixing transmission $T_{\uparrow \downarrow}$, and of their difference $A_{\uparrow \downarrow}=G_{\uparrow \downarrow}-T_{\uparrow \downarrow}$ in the units of $1 /\left(\mathrm{f} \Omega \mathrm{m}^{2}\right)$; and (b) magnitude of the second harmonic voltage response as a function of the free layer thickness in the $\mathrm{Cu}-\mathrm{Ni}(8) / \mathrm{Cu}(5) / \mathrm{Ni}(d) / \mathrm{Cu}(5) / \mathrm{Ni}(8)-\mathrm{Cu}$ valve with disordered interfaces of the central magnetic layer.

and present the main contribution to spin torque. In contrast to $\mathrm{Co} / \mathrm{Cu}$ spin valves, $\operatorname{Im} G_{\uparrow \downarrow}$ remains nonzero and thus $U_{2 \omega}$ also oscillates around a nonzero value. The disorder slightly reduces the TSPL which now can be estimated from the second harmonic voltage as equal to about 20 atomic planes.

\section{Au/Fe double spin valve}

Finally, let us consider a spin valve consisting of iron and gold layers. More precisely, we assume the structure $\mathrm{Au}-\mathrm{Co}(8) / \mathrm{Au}(5) / \mathrm{Fe}(d) / \mathrm{Au}(5) / \mathrm{Co}(8)-\mathrm{Au}$. The bcc lattice of $\mathrm{Fe}$ is well matched (when rotated by $45 \mathrm{deg}$ ) to Au's fcc along the [001] interface. The perfect lattice match was assumed in $a b$ initio calculations with the lattice constants related by $a_{\mathrm{Au}}=$ $\sqrt{2} a_{\mathrm{Fe}}=4.053 \AA$. The distance at $\mathrm{Au} \mid \mathrm{Fe}$ interface was chosen so as to satisfy the local space-filling condition of ASA approximation, with the atomic spheres radii set to the bulk values.

Figure 9 shows the imaginary parts of the mixing parameters of the central magnetic Fe layer sandwiched between $\mathrm{Au}$ nonmagnetic films, together with the second harmonic response $U_{2 \omega}$. In this case, we do not see any pronounced initial decay of $\operatorname{Im} A_{\uparrow \downarrow}$ at smaller $N$. $\operatorname{Im} A_{\uparrow \downarrow}$ mostly oscillates around zero with damped amplitude, and so does also the second harmonic voltage. In turn, Fig. 10 shows the analogical comparison but for the spin valve with interfacial disorder at the free magnetic layer.

From the numerical results follows that for the system with clean interfaces the TSPL is relatively long and can be

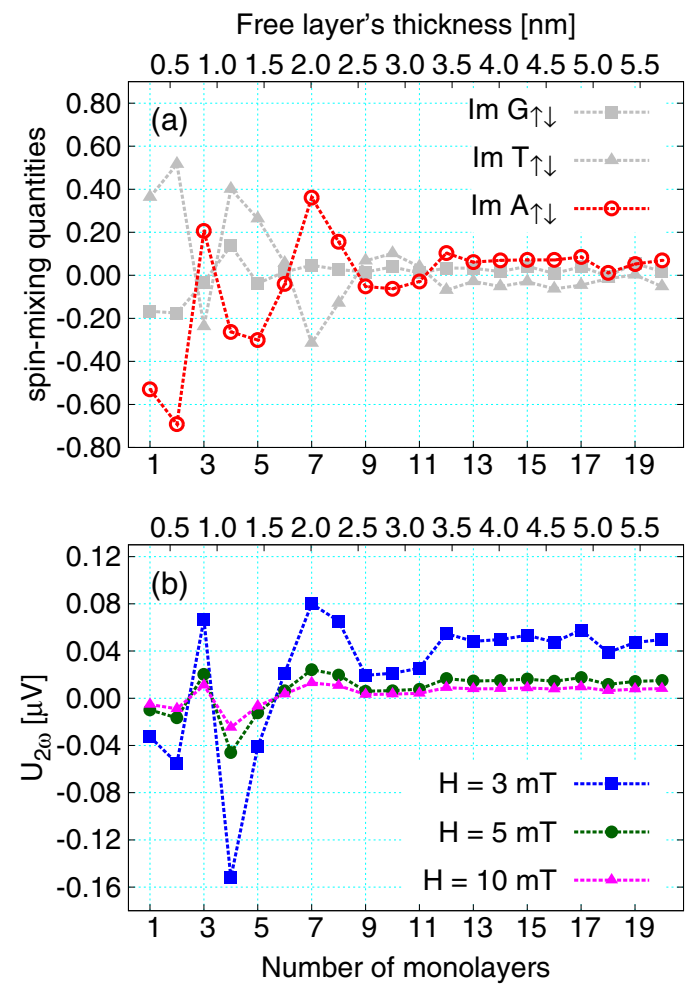

FIG. 9. (a) Imaginary parts of the mixing conductance $G_{\uparrow \downarrow}$, mixing transmission $T_{\uparrow \downarrow}$, and of their difference $A_{\uparrow \downarrow}=G_{\uparrow \downarrow}-T_{\uparrow \downarrow}$ in the units of $1 /\left(\mathrm{f} \Omega \mathrm{m}^{2}\right)$; and (b) magnitude of the second harmonic voltage response as a function of the free layer thickness in the $\mathrm{Au}-\mathrm{Co}(8) / \mathrm{Au}(5) / \mathrm{Fe}(d) / \mathrm{Au}(5) / \mathrm{Co}(8)-\mathrm{Au}$ valve with clean (ordered) interfaces.

estimated to be about 8 atomic monolayers. The impact of disorder on the transport parameters is relatively strong and no clear conclusions on TSPL can be drawn. However, it seems that the TSPL is the presence of disorder is comparable with that in systems with clean interfaces.

\section{SUMMARY AND CONCLUSIONS}

In this paper we have presented a theoretical analysis of transverse spin transport through thin magnetic layers, including a few to over a dozen atomic monolayers. First, we have shown that the second harmonic voltage is proportional to the out-of-plane torque. This means that in ultrathin magnetic layers, $U_{2 \omega}$ is proportional to the imaginary part of $A_{\uparrow \downarrow}$, defined as $A_{\uparrow \downarrow}=G_{\uparrow \downarrow}-T_{\uparrow \downarrow}$. Although this relation seems to be straightforward, $U_{2 \omega}$ is a complicated function of more parameters, including also the spin valve magnetoresistance. In order to demonstrate usefulness of the second harmonic measurements for estimation of the TSPL in magnetic free layers, we studied $U_{2 \omega}$ numerically in the spin-dependent diffusive transport model, treating the free layer as a ballistic magnetic scatterer.

In general, it is assumed that the transmission of transverse spin current components through a ferromagnet is suppressed to zero rather quickly, and for a good measure of the spin penetration one should take into account also the mixing transmission $T_{\uparrow \downarrow}$. On the other hand, as shown in our ab initio calculations, as well as in earlier studies [34], the decay 
Free layer's thickness [nm]
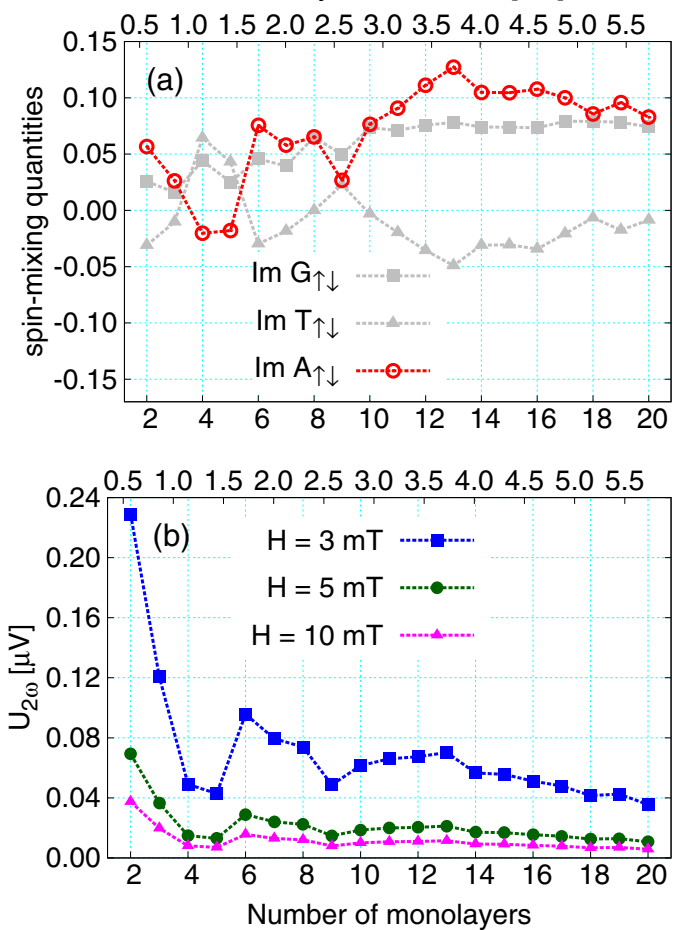

FIG. 10. (a) Imaginary parts of the mixing conductance $G_{\uparrow \downarrow}$, mixing transmission $T_{\uparrow \downarrow}$, and of their difference $A_{\uparrow \downarrow}=G_{\uparrow \downarrow}-T_{\uparrow \downarrow}$ in the units of $1 /\left(\mathrm{f} \Omega \mathrm{m}^{2}\right)$; and (b) magnitude of the second harmonic voltage response as a function of the free layer thickness in the $\mathrm{Au}-$ $\mathrm{Co}(8) / \mathrm{Au}(5) / \mathrm{Fe}(d) / \mathrm{Au}(5) / \mathrm{Co}(8)-\mathrm{Au}$ valve with disordered interfaces of the central magnetic layer.

of $T_{\uparrow \downarrow}$ components with thickness hardly can be described as exponential, but it is rather an algebraic function of the thickness. However, in the case of algebraic functions one cannot define a simple decay scale. Moreover, it is shown that due to quantum effects, $T_{\uparrow \downarrow}$ might oscillate and reach again considerable values at higher thicknesses. These oscillations in magnitude of $T_{\uparrow \downarrow}$ components are, however, usually suppressed due to interface disorder. Thus, one of the possible definition of TSPL is the thickness of the magnetic layer, where $T_{\uparrow \downarrow}$ components approaches zero for the first time. When the oscillations are well resolved and damped exponentially, one can define TSPL unequivocally. If the magnitudes of both $T_{\uparrow \downarrow}$ components decay in the same manner, one might focus on just one of them. Moreover, if, as is often the case, the values of $G_{\uparrow \downarrow}$ components decrease more sharply with the free layer thickness than that of $T_{\uparrow \downarrow}$, then one can use $\operatorname{Im} A_{\uparrow \downarrow}$ to estimate the TSPL. This opens the possibility to utilize a measurement of the out-of-plane STT to estimate the TSPL. Thus, the second harmonic voltage response appears as a helpful measure of the TSPL.

Consequently, we have studied the second harmonic response voltage as a potential method for TSPL measurement. We assumed the magnetic layer to be embedded as a free magnetic layer into a dual spin valve with antiparallel alignment of the fixed magnetic moments. In this model, the transport properties of the free magnetic layer are fully described by the channel conductances, mixing conductance, and mixing transmission. Calculating the spin torque acting
TABLE I. Bulk material parameters used for the layers.

\begin{tabular}{lccc}
\hline \hline Material & $\rho^{*}(\mu \Omega \mathrm{cm})$ & $\beta$ & $\lambda_{\text {sf }}(\mathrm{nm})$ \\
\hline $\mathrm{Co}$ & 5.1 & 0.51 & 60 \\
$\mathrm{Ni}$ & 41.5 & -0.35 & 3.3 \\
$\mathrm{Cu}$ & 0.5 & 0 & 350 \\
$\mathrm{Au}$ & 2.0 & 0 & 60 \\
\hline \hline
\end{tabular}

on the free magnetic layer and magnetoresistance of the spin valve, we evaluated the second harmonic response voltage as a function of the in-plane magnetic field orientation. We have shown a close correlation between $A_{\uparrow \downarrow}$ and the second harmonic voltage.

It is noteworthy that the proposed transport measurement method differs from the previously used methods employing spin pumping [16-18]. In the previous studies, the experiments were focused on the examination of the dominant real part of the spin mixing conductance, which depends just on the reflection from the N/FM interface only. In the current study, we consider the second harmonic voltage measurement, which gives us access to smaller effects of the spin mixing transmission by assessing the strength of the fieldlike torque. To conclude, from our estimations it follows that typical TSPL in transition-metal multilayers is of the order of a few atomic layers (from 4 up to 20), and thus an assumption of a rapid decay in the case of ultrathin free layers is not always justified. Knowledge of TSPL might be useful for the development of spin torque-based devices and for the choice of a proper model of spin transport.

\section{ACKNOWLEDGMENTS}

This research has been partly supported in frame of the Project NANOSPIN PSPB-045/2010 supported by a grant from Switzerland through the Swiss contribution to the enlarged European Union, and partly by the National Science Center in Poland as the Project No. DEC2012/04/A/ST3/00372. P.B. acknowledges support from the Czech Science Foundation (Grant No. 15-08740Y), and useful discussions with I. Turek and K. Carva.

\section{APPENDIX: BULK AND INTERFACE PARAMETERS}

Except for the ultrathin central magnetic layer, the spindependent electronic transport through the magnetic and nonmagnetic layers was described in the diffusive transport model [32,39]. In frame of this approach, transport is described separately for the bulk of magnetic layers and interfaces between the layers. In Tables I and II we show the bulk and interface transport parameters, respectively, which were

TABLE II. Interface material parameters used for the interfaces. $R^{*}$ is given in the units of $\mathrm{f} \Omega \mathrm{m}^{2}$ and the mixing conductance is given in $1 /\left(\mathrm{f} \Omega \mathrm{m}^{2}\right)$.

\begin{tabular}{llrcr}
\hline \hline Interface & \multicolumn{1}{c}{$R^{*}$} & \multicolumn{1}{c}{$\gamma$} & $\operatorname{Re} G_{\uparrow \downarrow}$ & $\operatorname{Im} G_{\uparrow \downarrow}$ \\
\hline $\mathrm{Cu} / \mathrm{Co}$ & 0.5 & 0.77 & 0.542 & 0.016 \\
$\mathrm{Cu} / \mathrm{Ni}$ & 0.612 & -0.52 & 0.820 & 0.060 \\
$\mathrm{Au} / \mathrm{Co}$ & 0.5 & 0.70 & 0.390 & 0.012 \\
\hline \hline
\end{tabular}


used in the numerical calculations presented in Sec. III. The interface parameters are analogical to those used to describe ultrathin free magnetic layer. The bulk transport parameters are the mean bulk resistivity $\rho^{*}$ and the bulk spin asymmetry parameter $\beta$, which allow us to write the bulk channel resistivities as $\rho_{\uparrow(\downarrow)}=2 \rho^{*}(1 \mp \beta)$. Moreover, $\lambda_{\mathrm{sf}}$ is the spin-flip length. The parameters were taken from Refs. [39,40].
[1] J. C. Slonczewski, J. Magn. Magn. Mater. 159, L1 (1996).

[2] L. Berger, Phys. Rev. B 54, 9353 (1996).

[3] M. D. Stiles and A. Zangwill, Phys. Rev. B 66, 014407 (2002).

[4] A. Brataas, A. D. Kent, and H. Ohno, Nat. Mater. 11, 372 (2012).

[5] A. D. Kent and D. C. Worledge, Nat. Nanotechnol. 10, 187 (2015).

[6] H.-S. Philip Wong and S. Salahuddin, Nat. Nanotechnol. 10, 191 (2015).

[7] M. N. Baibich, J. M. Broto, A. Fert, F. Nguyen Van Dau, F. Petroff, P. Etienne, G. Creuzet, A. Friederich, and J. Chazelas, Phys. Rev. Lett. 61, 2472 (1988).

[8] G. Binasch, P. Grunberg, F. Saurenbach, and W. Zinn, Phys. Rev. B 39, 4828 (1989).

[9] T. Valet and A. Fert, Phys. Rev. B 48, 7099 (1993).

[10] J. Xiao and G. E. W. Bauer, Phys. Rev. Lett. 108, 217204 (2012).

[11] L. Gravier, S. Serrano-Guisan, F. Reuse, and J.-P. Ansermet, Phys. Rev. B 73, 052410 (2006).

[12] M. Hatami, G. E. W. Bauer, Q. Zhang, and P. J. Kelly, Phys. Rev. Lett. 99, 066603 (2007).

[13] H. Yu, S. Granville, D. P. Yu, and J.-P. Ansermet, Phys. Rev. Lett. 104, 146601 (2010).

[14] Y. Tserkovnyak, A. Brataas, and G. E. W. Bauer, Phys. Rev. Lett. 88, 117601 (2002).

[15] M. Battiato, K. Carva, and P. M. Oppeneer, Phys. Rev. Lett. 105, 027203 (2010).

[16] A. Ghosh, S. Auffret, U. Ebels, and W. E. Bailey, Phys. Rev. Lett. 109, 127202 (2012).

[17] T. Taniguchi, S. Yakata, H. Imamura, and Y. Ando, IEEE Trans. Magn. 44, 2636 (2008).

[18] T. Taniguchi, S. Yakata, H. Imamura, and Y. Ando, Appl. Phys. Express 1, 031302 (2008).

[19] S. Zhang, P. M. Levy, and A. Fert, Phys. Rev. Lett. 88, 236601 (2002).

[20] A. Shpiro, P. M. Levy, and S. Zhang, Phys. Rev. B 67, 104430 (2003).

[21] Y. Tserkovnyak, A. Brataas, G. E. W. Bauer, and B. I. Halperin, Rev. Mod. Phys. 77, 1375 (2005).
[22] A. A. Kovalev, A. Brataas, and G. E. W. Bauer, Phys. Rev. B 66, 224424 (2002).

[23] P. Baláž, J. Barnaś, and J.-P. Ansermet, J. Appl. Phys. 113, 193905 (2013).

[24] J. Dubois and J.-P. Ansermet, Phys. Rev. B 78, 184430 (2008).

[25] H. Yu, J. Dubois, S. Granville, D. P. Yu, and J.-P. Ansermet, J. Phys. D: Appl. Phys. 42, 175004 (2009).

[26] A. A. Kovalev, G. E. W. Bauer, and A. Brataas, Phys. Rev. B 75, 014430 (2007).

[27] O. K. Andersen, O. Jepsen, and D. Glötzel, in Highlights of Condensed Matter Theory, International School of Physics 'Enrico Fermi', Varenna, Italy, edited by F. Bassani, F. Fumi, and M. P. Tosi (North-Holland, Amsterdam, 1985), pp. 59-176.

[28] I. Turek, V. Drchal, J. Kudrnovský, M. Šob, and P. Weinberger, Electronic Structure of Disordered Alloys, Surfaces and Interfaces (Kluwer, Boston, 1997).

[29] K. Xia, P. J. Kelly, G. E. W. Bauer, A. Brataas, and I. Turek, Phys. Rev. B 65, 220401 (2002).

[30] M. Zwierzycki, P. A. Khomyakov, A. A. Starikov, K. Xia, M. Talanana, P. X. Xu, V. M. Karpan, I. Marushchenko, I. Turek, G. E. W. Bauer et al., Phys. Status Solidi (b) 245, 623 (2008).

[31] A. Brataas, G. E. Bauer, and P. J. Kelly, Phys. Rep. 427, 157 (2006).

[32] J. Barnaś, A. Fert, M. Gmitra, I. Weymann, and V. K. Dugaev, Phys. Rev. B 72, 024426 (2005).

[33] P. Baláž, M. Gmitra, and J. Barnaś, Phys. Rev. B 80, 174404 (2009).

[34] M. Zwierzycki, Y. Tserkovnyak, P. J. Kelly, A. Brataas, and G. E. W. Bauer, Phys. Rev. B 71, 064420 (2005).

[35] K. Carva and I. Turek, Phys. Rev. B 76, 104409 (2007).

[36] L. Berger, J. Appl. Phys. 93, 7693 (2003).

[37] M. D. Stiles and J. Miltat, Spin Dynamics in Confined Magnetic Structures III (Springer, Berlin, Heidelberg, 2006), pp. 225-308.

[38] K. Carva and I. Turek, Phys. Status Solidi (a) 205, 1805 (2008).

[39] M. Gmitra and J. Barnaś, in Toward Functional Nanomaterials, edited by Z. Wang (Springer, Berlin, 2009), pp. 285-322.

[40] A. Reilly, W. Park, R. Slater, B. Ouaglal, R. Loloee, W. P. Pratt Jr., and J. Bass, J. Magn. Magn. Mater. 195, L269 (1999). 